\title{
Ethnicity and Renal Replacement Therapy
}

\section{John Feehally}

The John Walls Renal Unit, Leicester General Hospital \& Department of Infection, Immunity, \& Inflammation, University of Leicester, UK

\section{Correspondence to:}

Professor John Feehally, The John Walls Renal Unit, Leicester General Hospital, Gwendolen Road, Leicester LE5 4PW, UK. Phone: +44 116258 4132. Fax: +44 116 258 4764; E-mail: jf27@le.ac.uk 


\section{ABSTRACT}

There are significant ethnic variations in the incidence of kidney disease. White European populations appear to be uniquely protected compared to increased incidences of ESRD in indigent and migrant ethnic minority populations. This increase is partly explained by a high prevalence of diabetic nephropathy, but there is also increased susceptibility to a range of other renal diseases. The relative contributions of genetic, environmental, and fetal environmental factors to this susceptibility are not yet well understood.

Strategies for early detection and management of CKD to delay progression are particularly critical in countries where access to RRT is restricted.

In developed countries with wide availability of RRT, resources to provide dialysis will need to be increased in regions with substantial minority populations. There is apparently counterintuitive evidence that survival on dialysis is increased in many minority populations. Access to renal transplantation, both from deceased and living donors, is also restricted in many minority populations, and graft survival is often inferior.

Analysis of the explanations for these differences is complex because of the many confounding factors [for example cultural, social, and economic] which typically cosegregate with ethnicity. Nevertheless reduction of the varied and substantial inequities faced by ethnic minority populations with kidney disease is an important responsibility for the renal community

\section{$\underline{\text { Key Words }}$}

Ethnic minorities -Renal replacement therapy - Hemodialysis - Peritoneal dialysis Renal transplantation 


\section{Introduction}

People from indigenous or migrant ethnic minority populations have increased susceptibility to chronic kidney disease [CKD]. This offers many challenges:

- for researchers in understanding the complexes processes which underlie this susceptibility;

- for clinicians seeking to prevent or delay the progression of CKD in an individual patient, and optimising renal replacement therapy [RRT] when this is unavoidable,;

- for those responsible for organising, delivering and meeting the cost of the increased demands for RRT in areas serving minority populations.

While it is pragmatically necessary to make broad categories of ethnicity to purposes of epidemiology and clinical research in this field, it is also important to appreciate that within any grouping such as African or South Asian are populations with enormous variety of culture, religion, education, and socio-economic circumstances which makes complex the interpretation of causal relationships.

\section{Epidemiology}

Migration of populations across the world continues to increase. This has led to an increasing variety of ethnic minority populations in many countries, some of which also have minority indigenous populations. Minority populations are often geographically localised. For example, ethnic minorities are $9 \%$ of the UK 
population, but in the city of Leicester, the population originating from South Asia has rapidly increased to more than $50 \%$ of those living within the city boundary.

Such minority populations present challenges to health care. Even if they had no variations in disease prevalence, substantial language and cultural variations can provide additional demands on the health care system, and these are most difficult in long term conditions such as CKD. But in fact, there are many disease variations in minority populations, not least an increased incidence of type 2 diabetes and progressive CKD. Furthermore recent migrant populations typically have a younger age distribution than the indigenous population, so that diseases such as CKD, which increase in incidence with increasing age, will at first be underrepresented compared to the eventual population burden as the minority population ages.

The incidence of CKD is increased in many ethnic minority populations. There a is three- to fourfold increase in the incidence of end-stage renal disease (ESRD) in South Asian and African Caribbean populations in the UK [1], and there are similar increases in African Americans, Hispanics, and Native Americans in the US [2]. Other populations at increased risk include Aborigines, Maoris, and Pacific Islanders in Australasia. Most studies have used the onset of ESRD and acceptance for RRT as a means of case ascertainment, but this approach might underestimate the true incidence if patients have inequity of access to RRT. It is common to emphasize the increased susceptibility to ESRD in these ethnic minority populations; but perhaps we should instead consider that the white European population seems uniquely at low risk of renal disease. This decreased risk could have a genetic basis, or may reflect environmental factors, such as the very gradual urbanization in most 
Caucasian European populations compared to the very rapid recent urbanization in many developing countries.

In more homogeneous populations, the incidence of ESRD is even more strikingly increased. While the Pima Indians have a specific susceptibility to diabetic nephropathy [3], Native Americans of the Zuni Pueblo tribe--with an incidence of ESRD 18 times that of white Americans--have a broader susceptibility to CKD including diabetic nephropathy and glomerulonephritis [4]. The Aboriginal population in Tiwi Island, Northern Territory, where obesity and type 2 diabetes are endemic, has an ESRD incidence more than 2000/million population/year; comprising only $26 \%$ of the population of Northern Territory, Aborigines are $96 \%$ of those on RRT [5].

All these ethnic minority populations at increased risk of CKD share a marked increase in susceptibility to type 2 diabetes and an increased susceptibility among diabetics to ESRD. For example, data from Leicester, UK indicated a 13-fold increase in the risk of a South Asian diabetic developing ESRD compared to a white diabetic [6], the Pima Indians have a risk of ESRD 14 times that of the white US diabetic population [7], and there is also a marked increase in African American type 2 diabetics compared to whites. In many populations severe hypertension adds risk for ESRD. Current predictions suggest an increase in the prevalence of type 2 diabetes of at least $30 \%$ above current levels by the year 2025 in developed countries, but two- to threefold increases in prevalence in developing countries during the same period [8]. This predicted epidemic of ESRD in people with diabetes will be uncontainable unless effective preventive strategies are developed. 
Studies using requirement for RRT for case ascertainment will not distinguish susceptibility to the development of renal disease from an increased risk of progression to ESRD once nephropathy is established. In hypertensive blacks, there is long-established evidence of increased progression risk despite equivalent blood pressure control [9]. In CKD of other etiologies, evidence increasingly points to the increased risk of progression as the predominant explanation for increased ESRD. A US birth cohort analysis showed no difference between blacks and whites in the prevalence of CKD, yet five years later in the same cohort, a near fivefold increased risk of ESRD among the blacks was apparent [10]. Recent UK data also show no difference in the prevalence of early CKD between White and South Asian populations despite the marked excess of ESRD in South Asians.

\section{Pathogenesis}

Genetic susceptibility to renal diseases and their progression, susceptibility provoked by environmental factors, susceptibility induced by the effects of fetal environment, and socioeconomic influences all can contribute to susceptibility to CKD in ethnic minority populations [11].

One particular challenge is separating the influence of ethnicity from that of socio-economic disadvantage, two major factors which so often co-segregate. Socioeconomic disadvantage is itself associated with increased risk of ESRD [12], a complex interaction that might directly influence renal damage, be associated with damaging health behaviours, or influence the quality of health care of those with kidney disease. 


\section{Preventing progression to ESRD}

There is much work needed to unravel the contributions of these various pathogenic mechanisms, but in the meantime there are opportunities to prevent CKD in high risk populations, and to detect CKD early, intervening to delay its progression. If such opportunities are grasped, they may not only benefit the individual, but offer significant health system economies if they avoid the cost of RRT, and of course are critically important in health economies where RRT is unavailable or unaffordable.

But such opportunities to turn the tide of ESRD will not be grasped if those at risk are not engaged by the healthcare system nor are responsive to our recommendations. Minority populations may lack the awareness or health education to engage, and programmes which approach these problems must be designed with cultural sensitivity.

In the US, considerable evidence points to blacks having inferior access to health care. Blacks report receiving less information about health care and express more dissatisfaction with treatment [13]. Fewer black hypertensive patients have their blood pressure checked at least annually. These differences are reported at all income levels, although blood pressure control might be more unsatisfactory among blacks living in inner cities [14]. The only similar study in the UK shows a more reassuring trend for earlier referral of blacks with CKD compared to whites. [15].

\section{Outcome of renal replacement therapy in ethnic minority populations}


In healthcare settings where RRT is available, it is important that RRT be delivered with equity in ethnic minority populations. Proper assessment of equity requires evaluation of mortality, morbidity, and quality of life, if care is to be optimised.

In the US, mortality in patients receiving RRT (adjusted for socio-economic factors and comorbidity), is consistently lower in ethnic minorities [16]. The UK Renal Registry reports a significant reduction in one-year mortality for Blacks and South Asians on RRT [17]. In Hong Kong, survival of Chinese patients undergoing peritoneal dialysis (PD) is significantly better than that reported in other large PD studies involving predominantly white populations [18]. The reasons for these discrepant outcomes are likely to be complex; ethnicity itself might not be the explanation, and many other factors might introduce bias. For example, $80 \%$ of all dialysis patients in Hong Kong are undergoing PD compared to the predominance of HD in the US. A low patient acceptance also might alter the case mix; only $54 \%$ of Hong Kong Chinese identified as suitable for PD agreed to receive the treatment [19]. A low transplantation rate, as in Hong Kong, also might lead to younger, fitter patients remaining on $\mathrm{PD}$, thus improving the cohort outcome. The improved survival may also reflect survival advantage; the highest risk individuals having not survived to commence RRT.

Improved survival on haemodialysis is found in African Americans despite other factors expected to reduce survival including non-adherence with treatment regimens, increased use of dialysis catheters, and higher requirements for erythropoietin [20].

\section{Renal Transplantation}


Registry data from several parts of the world including the UK and the US show lower rates of transplantation in ethnic minorities which may represent inequity of access. Ethnic minorities are overrepresented in dialysis programs but less likely to be listed for deceased donor transplantation, wait longer, and are less likely actually to be transplanted [21]. Ethnic minority populations are more likely to receive poorly matched kidneys, since they are under-represented among deceased donors. Graft survival is also reduced in minorities in the US, and in both Blacks and South Asians in the UK [21, 22]. But the most disadvantaged patients of course are eligible patients who are never transplanted, whether with a deceased donor or living donor kidney.

Promotion of living donor transplantation is a key element in strategies for minimizing inequity of access to transplantation. A study from Baltimore, USA showed that a decade of active encouragement of volunteerism led to the same living donor transplant rate in blacks as whites, with equivalent patient and graft survival, and median waiting times below the national average [23]. Innovative approaches to living donor transplantation, for example, strategies aimed at transplanting across ABO incompatibility barriers, also might assume special importance. US physicians when asked why ethnic minority patients were referred less frequently for transplantation most commonly cited the lack of a potential live donor, patient preference, co-morbidity, or concern that patients did not complete necessary evaluations [24]. While living donor transplantation is the optimal RRT for many patients, donor selection needs special care in populations which have typically have a high incidence of familial renal disease. 
The shortage of deceased donors from ethnic minority populations is a continuing concern. In the UK, South Asians and Blacks only contribute $1.5 \%$ of the total donor pool yet comprise $6 \%$ of the general population and $19 \%$ of patients on the deceased donor transplant waiting list. The reasons underlying this shortfall have been extensively explored with these communities, and programs are in place to improve donor rates through awareness and education, although progress is slow.

An adverse effect of the relative difficulties in identifying appropriate deceased donors for minority populations has been the surge of 'transplant tourism' for those with resources to 'buy' a living unrelated donor kidney, a practice condemned by the international renal community through the Istanbul Declaration [25] .

\section{Quality of life on renal replacement therapy}

Tools for the evaluation of health-related quality of life are available for renal disease. However, these have typically been developed and tested in majority populations, and might not be sensitive to the cultural, religious, and social distinctions in ethnic minorities. Important differences also might emerge when questionnaire-based quality-of-life assessment relies on the use of health professionals, interpreters, and family members. These potential biases will not be infrequent, even in stable migrant populations, in which older people do not read or write in English or even in their native language.

Nevertheless, South Asians in the UK reported inferior quality of life compared to whites both on peritoneal dialysis and hemodialysis, and following renal 
transplantation [26]. However, health-related quality of life in the Dialysis Outcomes and Practice Patterns Study (DOPPS) was higher in ethnic minority patients [27]. The prominence of religious belief as a coping mechanism has been suggested as a possible explanation for this finding [28]; alternatively, in the US health care setting, a narrowing of the gap in access to good quality health care after the initiation of RRT might alter perception of quality of life.

\section{Public health implications}

Although exploration of the mechanisms that underlie susceptibility to renal disease in these populations is of the utmost importance for the future, there is a pressing worldwide health care challenge that will accelerate at a pace faster than fundamental research can head off. In the UK, the overall age-adjusted relative risk remains more than threefold in both the South Asian and African Caribbean populations, and this risk might be increasing. The younger age distribution of the ethnic minority population means even larger future increases in the demand for RRT. Estimates of need are based on the presumption that current acceptance rates are a true reflection of demand; this might not be the case if there is inequity of access to health care. Vigorous advocacy must continue if resources sufficient to deliver the required standards of care are to be made available where populations include large ethnic minorities. Indeed it must be recognised that the delivery of high-quality health care that is linguistically and culturally adequate for ethnic minorities will likely require additional resources compared to the resources needed for majority populations. Although intensive efforts at augmenting transplantation 
rates from both deceased and living donors must continue, a disproportionate requirement for dialysis facilities is unavoidable. In parallel, programmes for screening and early intervention must be introduced if the future RRT burden is to be restrained [29]. Priority also must be given to the long-term goals of understanding genetic and other factors influencing the susceptibility of ethnic populations to CKD if truly effective preventative strategies are to be achieved. 


\section{REFERENCES}

1. Lightstone L, Rees AJ, Tomson C, et al: High incidence of end-stage renal disease in Indo-Asians in the UK. Q J Med 88:191-195, 1995

2. U.S. Renal Data System, USRDS 2008 Annual Data Report: Atlas of Chronic Kidney Disease and End-Stage Renal Disease in the United States, National Institutes of Health National Institutes of Diabetes and Digestive and Kidney Diseases, Bethesda MD 2008. www.usrds.org/adr.htm

3. Nelson RG: Diabetic renal disease in transitional and disadvantaged populations. Nephrology 6: 9-17, 2001

4. Shah VO, Scavini M, Stidley C, et al: Epidemic of diabetic and non-diabetic renal disease among the Zuni Indians: the Zuni Kidney Project. J Am Soc Nephrol 14:1320-1329, 2003

5. Hoy W: Renal disease in Australian Aborigines. Nephrol Dial Transplant $15: 1293-1297,2000$

6. Burden AC, McNally PG, Feehally J, Walls J: Increased incidence of endstage renal failure secondary to diabetes mellitus in Asian ethnic groups in the United Kingdom. Diab Med 9:641-645, 1992 
7. Nelson RG, Newman JM, Knowler WC, et al: Incidence of end-stage renal disease in Type 2 (non-insulin-dependent) diabetes mellitus in Pima Indians. Diabetologia 31:730-736, 1988

8. King H, Aubert RE, Herman WH: Global burden of diabetes, 1995-2025: prevalence, numerical estimates, and projections. Diabetes Care 21:296-309, 1998

9. Rostand SG, Brown G, Kirk KA, et al: Renal insufficiency in treated essential hypertension. N Engl J Med 88:1696-1699, 1989

10. Hsu CY, Lin F, Vittinghoff E, Shlipak MG: Racial differences in the progression from chronic renal insufficiency to end-stage renal disease in the United States. J Am Soc Nephrol 14:2902-2907, 2003

11. Buck K, Feehally J: Diabetes and renal failure in Indo-Asians in the UK - a paradigm for the study of disease susceptibility. Nephrol Dial Transplant 12: $1555-1557,1997$

12. Cass A, Cunningham J, Snelling P, et al: Exploring the pathways leading from disadvantage to end-stage renal disease for indigenous Australians. Soc Sci Med 58:767-785, 2004 
13. Powe N: Nephrology Forum: To have and have not: Health and health care disparities in chronic kidney disease. Kidney Int 64:763-772, 2003

14. Kotchen JM, Shakoor-Abdullah B, Walker WE, et al: Hypertension control and access to health care in the inner city. Am J Public Health 88:16961699,1998

15. Steel J, Ellis P: Do demographic variables affect the timing of referral to a nephrologist? EDTNA ERCA J 28:185-187, 2002

16. Frankenfield DL, Krishnan SM, Ashby VB et al. Differences in mortality among Mexican-American, Puerto Rican, and Cuban-American dialysis patients in the United States. Am J Kidney Dis. 2009 Apr;53:647-57

17. Roderick P, Byrne C, Casula A et al. Survival of patients from South Asian and Black populations starting renal replacement therapy in England and Wales. Nephrol Dial Transplant. 2009 Jul 21. [Epub ahead of print]

18. Li PKT, Chow KM, Szeto CC: Is there a survival advantage in Asian peritoneal dialysis patients? Int J Artif Org 26:363-372, 2003

19. Lo WK, Li FK, Choy CBY et al: A retrospective survey of attitudes toward acceptance of peritoneal dialysis in Chinese end-stage renal failure patients 
in Hong Kong - from a cultural point of view. Perit Dial Int 3:S318-S321, 2001

20. Obrador GT. Anemia of chronic kidney disease and end-stage renal disease: are there unique issues in disadvantaged populations? Ethn Dis. 2009;19(1 Suppl 1):S1-52-5.

21. Rudge C, Johnson RJ, Fuggle SV et al. Renal transplantation in the United Kingdom for patients from ethnic minorities. Transplantation. 2007 ;83:1169-73

22. Feyssa E, Jones-Burton C, Ellison G, Philosophe B, Howell C Racial/ethnic disparity in kidney transplantation outcomes: influence of donor and recipient characteristics. J Natl Med Assoc. 2009 Feb;101:111-5.

23. Foster CE, Philosophe B, Schweistzer EJ, et al: A decade of experience with renal transplantation in African Americans. Ann Surg 236:794-804, 2002

24. Ayanian JZ, Cleary PD, Keogh JH, et al: Physicians' beliefs about racial differences in referral for renal transplantation. Am J Kidney Dis 43:350357,2004

25, The Declaration of Istanbul on organ trafficking and transplant tourism. International Summit on Transplant Tourism and Organ Trafficking. Kidney Int. 2008 Oct;74(7):854-9. 
26 Bakewell A, Higgins R, Edmunds ME: Does ethnicity influence perceived quality of life of patients on dialysis and following renal transplant? Nephrol Dial Transplant 16:1395-1401, 2001

27 Lopes AA, Bragg-Gresham JL, Satayathum S et al: Health-related quality of life and associated outcomes among hemodialysis patients of different ethnicities in the United States: The Dialysis Outcomes and Practice Patterns Study. Am J Kidney Dis 41:605-615, 2003

28 Thomas CJ: The context of religiosity, social support and health locus of control: implications for the health-related quality of life of African-American hemodialysis patients. J Health Soc Policy $16: 43-54,2002$

29 Lightstone L: Preventing kidney disease: the ethnic challenge. National Kidney Research Fund 2001. ISBN 1-904227-00-7. 